\title{
Associação entre o aleitamento materno, introdução alimentar e desenvolvimento neuropsicomotor nos primeiros seis meses de vida
}

\author{
Associations between breastfeeding, \\ nourishing introduction and \\ neuropsychomotor development \\ in the first six months of life
}

\section{Asociación entre la lactancia materna, la introducción de alimentos y el desarrollo neuropsicomotor en los primeros seis meses de vida}

\author{
Taisa Ribeiro de Souza Oliveira* \\ Larissa Santos Souza* \\ Rodrigo Dornelas* \\ Danielle Ramos Domenis* \\ Kelly da Silva* \\ Raphaela Barroso Guedes-Granzotti*
}

\section{Resumo}

Introdução: o aleitamento materno tem função primordial para o desenvolvimento nutricional, emocional, físico, do sistema motor oral, assim como no desenvolvimento neuropsicomotor infantil. Objetivo: investigar a associação do desenvolvimento neuropsicomotor, da introdução alimentar com o

*Universidade Federal de Sergipe, Aracaju, SE, Brasil.

Contribuições dos autores:

TRSO: coleta, tabulação dos dados e redação do manuscrito.

LSS: coleta, tabulação dos dados e redação do manuscrito.

RD: interpretação dos dados e redação do manuscrito.

DRD: idealização e orientação do estudo, redação do manuscrito.

KS: interpretação dos dados e redação do manuscrito.

RBGG: idealização e orientação do estudo, interpretação dos dados e redação do manuscrito.

E-mail para correspondência: Raphaela Granzotti - raphaelabgg@gmail.com

Recebido: 26/09/2016

Aprovado: 03/05/2017 
aleitamento materno de lactentes nos primeiros seis meses de vida. Método: 16 lactentes, sem fatores de risco para atraso no desenvolvimento, foram acompanhados mensalmente desde a maternidade até os seis meses para a avaliação do desenvolvimento neuropsicomotor por meio do Teste de Triagem Denver II e da rotina alimentar e hábitos orais por meio de um questionário. Resultados: no primeiro mês, $87,5 \%$ dos lactantes foram amamentados em seio materno, mas em $62,5 \%$ houve a introdução precoce de chá, água e outros leites e, $68,7 \%$ apresentavam hábitos orais. No sexto mês $18,7 \%$ realizavam aleitamento materno exclusivo, $43,75 \%$ aleitamento misto e $37,5 \%$ faziam uso de leite artificial, sendo que $84,6 \%$ já haviam introduzido sólidos. 56,25\% mantinham os hábitos orais. Quanto à avaliação do desenvolvimento, na maternidade, todos apresentaram respostas adequadas. No sexto mês na área pessoal social, $93,75 \%$ apresentavam desenvolvimento adequado, e 6,25\% estavam avançadas. No motor fino adaptativo, 87,5 $\%$ adequado, $6,25 \%$ avançados e $6,25 \%$ apresentavam risco. Na linguagem $100 \%$ estavam adequadas à idade e, no motor grosseiro, $31,25 \%$ estavam adequadas e $68,75 \%$ avançadas. Conclusão: os lactentes que permaneceram em aleitamento materno apresentaram um melhor desenvolvimento neuropsicomotor e uma menor incidência de hábitos orais deletérios, não havendo diferença quanto à introdução alimentar.

Palavras-chave: Aleitamento materno; Desenvolvimento Infantil; Cuidado da criança; Fonoaudiologia.

\section{Abstract}

Introduction: breastfeeding plays a fundamental role for nutritional, emotional, physical, oral motor system, as well as neuropsychomotor child development. Objective: to investigate the association of neuropsychomotor development, and nourishing introduction with breastfeeding in the first six months of life. Method: 16 infants without risk factors for developmental delay were monitored monthly from motherhood to six months for the evaluation of neuropsychomotor development through the Screening Test Denver II and eating routine and oral habits through a questionnaire. Results: in the first month, $87.5 \%$ of infants were breastfed, but $62.5 \%$ had early introduction of tea, water and other milks and $68.7 \%$ had oral habits. In the sixth month $18.7 \%$ had exclusive breastfeeding, $43.75 \%$ mixed feeding and $37.5 \%$ were using artificial milk, and $84.6 \%$ had introduced solids. $56.25 \%$ had oral habits. All the infants had adequate development in the evaluation while their stay in the maternity. In the sixth month in social personal area $93.75 \%$ had adequate development, and $6.25 \%$ were advanced. In adaptive fine motor, $87.5 \%$ suitable, advanced $6.25 \%$ and $6.25 \%$ were at risk. In the language $100 \%$ were age appropriate and in gross motor $31.25 \%$ were adequate and $68.75 \%$ advanced. Conclusion: the children who remained breastfed showed a better neuropsychomotor development and a lower incidence of deleterious oral habits, with no difference in nourishing introduction.

Keywords: Breast Feeding; Child Development; Child Care; Speech, Language and Hearing Sciences.

\section{Resumen}

Introducción: la lactancia materna tiene un papel fundamental para el desarrollo nutricional, emocional y físico del sistema motor oral, así como para el desarrollo neuropsicomotor infantil. Objetivo: investigar la asociación entre desarrollo neuropsicomotor y la introducción de alimentos con lactancia materna en lactantes, durante los primeros seis meses de vida. Método: 16 lactantes sin factores de riesgo de retraso en el desarrollo, fueron controlados mensualmente de la maternidad hasta los seis meses, para la evaluación del desarrollo neuropsicomotor a través de la Prueba de Tría de Denver II y de la rutina alimentar y hábitos orales, a través de un cuestionario. Resultados: en el primero mes, el 87,5\% de los lactantes fueron amamantados al seno, pero en el 62,5\% hubo introducción temprana de té, agua y otras leches y $68,7 \%$ presentaban hábitos orales. En el sexto mes, el 18,7\% tenían exclusivamente la lactancia materna, el 43,75\% lactancia mixta y el 37,5\% utilizaban la leche artificial. El 84,6\% ya había introducido sólidos. El 56.25\% mantenía hábitos orales. En la evaluación del desarrollo, en la maternidad, todos presentaron respuestas adecuadas. En el sexto mes, en el área personal social, 93,75\% presentaban un desarrollo adecuado, y 6,25\% estaban avanzados. En la motricidad fina adaptativa, el 87,5\% se mostró 
adecuado, 6,25\% avanzados y 6,25\% presentaron riesgo. En el lenguaje el 100\% estaban adecuados para la edad y en la motricidad gruesa, el 31,25\% estaban adecuados y el $68.75 \%$ avanzados. Conclusión: los lactantes que permanecieron en la lactancia materna presentaron un mejor desarrollo neuropsicomotor y una menor incidencia de los hábitos orales perjudiciales, sin diferencias en cuanto a la introducción de alimentos.

Palabras claves: Lactancia materna; Desarrollo Infantil; Cuidado del Niño; Fonoaudiología.

\section{Introdução}

A Organização Mundial da Saúde (OMS) preconiza o aleitamento materno exclusivo (AME) nos primeiros seis meses de vida considerando que as condições nutritivas e imunológicas do leite materno não podem ser substituídas por nenhum outro produto natural ou sintetizadas, sendo também desnecessário a suplementação do leite materno com água, chás ou qualquer outro alimento complementar durante este período ${ }^{1}$.

Estudos demonstraram ${ }^{2,3}$ também a associação positiva entre o aleitamento materno (AM) e um melhor desenvolvimento não só na infância, mas também na adolescência e vida adulta, fornecendo evidências de que o leite materno é o alimento "padrão ouro" para o desenvolvimento do cérebro. Isso porque vários nutrientes e componentes do leite materno desenvolvem o cérebro tanto pela ação bioquímica e funcional assim como por meio de mudanças no sistema sensorial que afetam o seu desenvolvimento.

Além dos aspectos citados anteriormente, o AM também é importante para a correta maturação e crescimento das estruturas do sistema estomatognático (lábios, língua, mandíbula, maxila, bochechas, palato mole e duro, soalho da boca, musculatura oral e arcadas dentárias), mantendo-as aptas para exercer o desenvolvimento da musculatura facial, estimulando o desenvolvimento de diversas funções fisiológicas como sucção, deglutição, mastigação, respiração e falaª .

Entretanto, além dos fatores biológicos, o contexto socioeconômico, cultural e o apoio às lactantes em suas singularidades aparecem como desafios a serem enfrentados para o sucesso da amamentação sendo que as taxas de AM no Brasil, em especial as de amamentação exclusiva, ainda estão abaixo do recomendado. Apesar disso, essa taxa vem aumentando como consequência do desenvolvimento de ações como as Enfermarias Canguru para assistência de crianças prematuras e de baixo peso, a implantação de Hospitais Amigos da
Criança, a formação de grupos de apoio e incentivo ao $\mathrm{AM}$ visando seguir as orientações da $\mathrm{OMS}^{1,4,5}$.

Dentre os principais obstáculos para a prática do AME podemos citar a falta de conhecimento e conscientização da população em geral, dos profissionais de saúde e dos gestores; condutas inapropriadas e pouca qualificação dos profissionais de saúde; aspectos culturais; falta de confiança/ baixa estima da mãe; problemas com a mama e mamilo; falta de apoio ou suporte familiar; trabalho da mulher; além do marketing de formulas lácteas infantis ${ }^{3,4}$. As dificuldades presentes no processo do aleitamento estão geralmente relacionadas à falta de acompanhamento e informação coesa de acordo com suas necessidades, o que evidencia a carência da promoção, proteção e apoio da amamentação por parte dos profissionais de saúde ${ }^{6}$.

Todo profissional da área da saúde precisa estar preparado para prestar uma assistência eficaz, solidária, integral e contextualizada, que respeite o saber e a história de vida de cada mulher e que a ajude a superar medos, dificuldades e inseguranças ${ }^{7}$. Nesse contexto, cada vez mais o fonoaudiólogo vem atuando nos assuntos relacionados à amamentação, contribuindo com as equipes interdisciplinares para a promoção da saúde materno-infantil, estimulando o desenvolvimento neuropsicomotor adequado, prevenindo a instalação de hábitos orais deletérios e orientando quanto à importância do AME até os seis meses de vida.

Diante do exposto e da influência que o AM exerce sobre o desenvolvimento infantil é de extrema importância entender os aspectos que podem influenciar o sucesso ou não da amamentação. Sendo assim, o objetivo deste estudo foi investigar a associação do desenvolvimento neuropsicomotor, da introdução alimentar com o aleitamento materno de lactentes nos primeiros seis meses de vida.

\section{Metodologia}

Trata-se de um estudo quantitativo, longitudinal e exploratório. A pesquisa faz parte de um projeto piloto que irá acompanhar o desenvolvi- 
mento de lactentes em diversas áreas da saúde por um período de um ano. Foi aprovado pelo CAAE 42167415800005546 da instituição de origem e é baseado na Resolução 466/2012 do Conselho Nacional de Saúde. Os responsáveis assinaram o Termo de Consentimento Livre e Esclarecido (TCLE), respeitando assim a tomada de decisão livre e espontânea em participar do projeto.

A amostra desta pesquisa foi constituída por 16 lactentes sendo que os critérios de inclusão foram: bebês nascidos a termo de parto sem intercorrências, com 24 a 48 horas de vida, de mães maiores de 18 anos e residentes na zona urbana do município. A amostra foi coletada na Maternidade Municipal no período de setembro de 2015 a fevereiro de 2016.

As avaliações foram realizadas em sete momentos diferentes, uma ainda na maternidade e as outras em visitas domiciliares mensais até o sexto mês de vida do lactente para aplicação de um questionário sobre rotina alimentar e hábitos orais, avaliação do desenvolvimento neuropsicomotor e orientações quanto à amamentação, introdução de alimentação complementar, desenvolvimento neuropsicomotor e estratégias para estimulá-lo.

As visitas foram realizadas por duas discentes do curso de Fonoaudiologia treinadas pela docente orientadora do trabalho, que agendavam previamente por telefone com a mãe, em horário que esta considerasse mais conveniente e, na semana em que o lactente completava mais um mês de vida. Os instrumentos utilizados foram os seguintes instrumentos:

Questionário sobre a rotina alimentar e hábitos orais (Anexo): para conhecer a rotina alimentar e os hábitos orais utilizou-se um questionário elaborado pelas pesquisadoras com 18 questões, divididas nos seguintes itens: informações pessoais, dados socioeconômicos, informações sobre a saúde e acompanhamento médico do lactente e por último dados sobre aleitamento, alimentação e hábitos orais. A mãe respondeu às questões verbalmente e as mesmas foram gravadas e anotadas pelo pesquisador. Alguns itens foram preenchidos apenas na primeira, enquanto que outros como os dados sobre alimentação e saúde foram preenchidos em todas as visitas, conforme descrito no questionário.

Para classificação do tipo de aleitamento materno foram utilizadas as definições adotadas pela Organização Mundial da Saúde $(\mathrm{OMS})^{8}$ e reconhecidas no mundo inteiro sendo:
- Aleitamento materno exclusivo (AME): quando a criança recebe somente leite materno, direto da mama ou ordenhado, ou leite humano de outra fonte, sem outros líquidos ou sólidos, com exceção de gotas ou xaropes contendo vitaminas, sais de reidratação oral, suplementos minerais ou medicamentos.

- Aleitamento materno predominante (AMP): quando a criança recebe, além do leite materno, água ou bebidas à base de água (água adocicada, chás, infusões), sucos de frutas e fluidos rituais.

- Aleitamento materno (AM): quando a criança recebe leite materno (direto da mama ou ordenhado), independentemente de receber ou não outros alimentos.

- Aleitamento materno complementado (AMC): quando a criança recebe, além do leite materno, qualquer alimento sólido ou semissólido com a finalidade de complementá-lo, e não de substituí-lo. Nessa categoria a criança pode receber, além do leite materno, outro tipo de leite, mas este não é considerado alimento complementar.

- Aleitamento materno misto ou parcial (AMM): quando a criança recebe leite materno e outros tipos de leite.

Avaliação do desenvolvimento Neuropsicomotor: para a avaliação do desenvolvimento neuropsicomotor foi utilizado o Teste de Triagem de Desenvolvimento de Denver II $^{9}$. O teste é composto de 125 itens distribuídos em 4 áreas do desenvolvimento: a) Pessoal-Social: aspectos da sociabilidade da criança dentro e fora do ambiente familiar; b) Motor Fino Adaptativo: coordenação viso-manual, manipulação de pequenos objetos; c) Linguagem: emissão de som, capacidade de reconhecer, entender e usar a linguagem e; d) Motor grosseiro: controle motor corporal, sentar, caminhar, pular e os demais movimentos realizados pela musculatura ampla.

O resultado de cada uma das áreas avaliadas foi considerado como normal, de risco, avançado e não testável de acordo com a interpretação de cada item avaliado, seguindo o seguinte critério: normal, quando não ocorreram atrasos ou, no máximo, um cuidado; avançado quando não ocorreram atrasos ou cuidado e a criança passou em itens à frente da linha da idade; risco de atraso, quando ocorreram dois ou mais cuidados ou um ou mais atrasos; e não testável quando a criança recusou-se a realizar um ou mais itens. 
Após a última visita, no sexto mês, para associação entre o tipo de aleitamento, o desenvolvimento neuropsicomotor e a introdução alimentar os lactentes foram divididos em dois grupos, sendo o G1 constituído por dez lactantes que mantiveram AM e o G2 constituído por seis lactentes com desmame precoce (DP).

Os resultados foram tabulados no software de planilha eletrônica Excel (pacote Microsoft ${ }^{\circledR}$ Office). Não foi realizado analise estatística inferencial devido ao número reduzido de bebês em cada grupo. Na estatística descritiva, os dados categóricos foram resumidos por meio de frequência absoluta (n) e relativa (\%) ao total de pacientes em cada grupo estudado.

\section{Resultados}

A média de idade das mães foi 26,8 anos ( \pm $5,7)$. Com relação ao nível socioeconômico das famílias a média da renda familiar per capita, foi de $\mathrm{R} \$ 499.82$, sendo que seis mães optaram por não responder. A média de indivíduos por residência foi 3,69 e a média de filhos por família foi de 1,94. Quanto à profissão das mães é possível observar na Tabela 1 que a maioria era dona de casa e lavradora. Na Tabela 1, além das informações quanto às variáveis socioeconômicas é possível observar informações referentes à gestação e ao parto.

$\mathrm{Na}$ avaliação do desenvolvimento neuropsicomotor, ainda na maternidade, todos os recém-nascidos apresentaram respostas normais em todas as áreas avaliadas. $\mathrm{Na}$ Tabela 2 é possível verificar, mês a mês, os resultados da triagem do desenvolvimento neuropsicomotor em todas as áreas avaliadas.

Com relação ao tipo de aleitamento $62,5 \%$ mantiveram o AM até os seis meses, entretanto em apenas $18,7 \%$ foi o aleitamento mantido o AME. O DP ocorreu em $37,7 \%$ a partir do terceiro mês e já no primeiro mês $68,7 \%$ já haviam introduzido algum tipo de líquido. Até o sexto mês nenhuma mãe introduziu alimentos sólidos, contudo os alimentos pastosos começaram a ser introduzidos a partir do quarto mês. A presença de hábitos orais foi observada em $68,7 \%$ dos lactentes no primeiro mês, enquanto que no sexto mês foi de $56,3 \%$, sendo prevalente o uso de chupetas (Tabela 2). Em todos os casos em que foi introduzido líquido, seja água, leite, chá ou suco, foi utilizada a mamadeira sendo a sua maioria, $70 \%$, com bico ortodôntico.
Tabela 1. Número e percentual dos dados socioeconômicos e gestacionais

\begin{tabular}{lc}
\hline Variável & $\mathbf{n}(\%)$ \\
\hline Número total de lactentes & $16(100)$ \\
Sexo masculino & $9(56,3)$ \\
Sexo feminino & $7(43,7)$ \\
\hline Escolaridade das mães & \\
Ensino fundamental incompleto & $6(37,5)$ \\
Ensino médio incompleto & $5(31,25)$ \\
Ensino superior completo & $2(12,5)$ \\
Ensino médio completo & $1(6,25)$ \\
Ensino fundamental completo & $1(6,25)$ \\
Sem instrução & $1(6,25)$ \\
\hline Profissão das mães & \\
Dona de casa & $5(31,25)$ \\
Lavradora & $5(31,25)$ \\
Estudante & $1(6,25)$ \\
Recepcionista & $1(6,25)$ \\
Costureira & $1(6,25)$ \\
Administradora & $1(6,25)$ \\
Cabeleireira & $1(6,25)$ \\
Farmacêutica & $1(6,25)$ \\
\hline Semanas de gestação & $14(87,5)$ \\
Entre 38 a 42 semanas & $2(12,5)$ \\
Menos de 38 semanas & $1(6,25)$ \\
Intercorrência gestacional & \\
\hline Tipo de parto & $10(62,5)$ \\
Cesárea & $16(100)$ \\
Normal & \\
Sem intercorrências durante o parto & \\
& \\
&
\end{tabular}

Considerando as avaliações do sexto mês e dividindo os lactentes em dois grupos, sendo o G1 constituído por dez lactantes que mantiveram AM e o G2 constituído por seis lactentes com DP é possível observar que, com relação à via de parto, no G1 $40 \%$ foi parto normal e $60 \%$ cesárea; já no G2 33,3\% foi parto normal e $66,7 \%$ cesárea.

Ao se comparar os hábitos orais observa-se que $70 \%$ do G1 não adquiriram hábitos orais, enquanto $100 \%$ do G2 possuíam hábitos orais. Quanto ao desenvolvimento neuropsicomotor foi observado um melhor desempenho no G1 já que $70 \%$ encontravam-se avançado para a idade em pelo menos uma das áreas averiguadas, $20 \%$ tinha o desenvolvimento normal e $10 \%$ apresentava risco de atraso. Com relação ao G2 50\% estavam com o desenvolvimento normal e 50\% avançado. 
Tabela 2. Número e percentual dos resultados do DENVER II, aleitamento, introdução alimentar e hábitos orais em cada uma das visitas.

\begin{tabular}{|c|c|c|c|c|c|c|}
\hline \multirow{2}{*}{ Avaliação } & $1^{\circ}$ mês & $2^{\circ}$ mês & $3^{\circ}$ mês & $4^{\circ}$ mês & $5^{\circ}$ mês & $6^{\circ}$ mês \\
\hline & n (\%) & n (\%) & n (\%) & n (\%) & n (\%) & n (\%) \\
\hline \multicolumn{7}{|l|}{ DENVER/ PS } \\
\hline Normal & $16(100)$ & $15(93,7)$ & $15(93,7)$ & $8(50,0)$ & $15(93,7)$ & $15(93,7)$ \\
\hline Avançado & 0 & $1(6,3)$ & $1(6,3)$ & $8(50,0)$ & 0 & $1(6,3)$ \\
\hline Risco & 0 & 0 & 0 & 0 & $1(6,3)$ & 0 \\
\hline Não testável & 0 & 0 & 0 & 0 & 0 & 0 \\
\hline \multicolumn{7}{|l|}{ DENVER/ MFA } \\
\hline Normal & $16(100)$ & $11(68,8)$ & $14(87,5)$ & $7(43,7)$ & $7(43,7)$ & $14(87,5)$ \\
\hline Avançado & 0 & $3(18,7)$ & $2(12,5)$ & $7(43,7)$ & $8(50,0)$ & $1(6,3)$ \\
\hline Risco & 0 & $2(12,5)$ & 0 & $2(12,5)$ & $1(6,3)$ & $1(6,3)$ \\
\hline Não testável & 0 & 0 & 0 & 0 & 0 & 0 \\
\hline \multicolumn{7}{|l|}{ DENVER/ LGG } \\
\hline Normal & $16(100)$ & $14(87,5)$ & $14(87,5)$ & $12(75,0)$ & $14(87,5)$ & $16(100)$ \\
\hline Avançado & 0 & $2(12,5)$ & $1(6,3)$ & $3(18,75)$ & $1(6,3)$ & 0 \\
\hline Risco & 0 & 0 & $1(6,3)$ & $1(6,3)$ & $1(6,3)$ & 0 \\
\hline Não testável & 0 & 0 & 0 & 0 & 0 & 0 \\
\hline \multicolumn{7}{|l|}{ DENVER/ MG } \\
\hline Normal & $14(87,5)$ & $14(87,5)$ & $12(75,0)$ & $12(75,0)$ & $9(56,3)$ & $5(31,3)$ \\
\hline Avançado & $2(12,5)$ & $2(12,5)$ & $3(18,7)$ & $3(18,7)$ & $6(37,5)$ & $11(68,7)$ \\
\hline Risco & 0 & 0 & $1(6,3)$ & 0 & $1(6,3)$ & 0 \\
\hline Não testável & 0 & 0 & 0 & $1(6,3)$ & 0 & 0 \\
\hline \multicolumn{7}{|c|}{ Tipo de Aleitamento } \\
\hline AME & $5(31,3)$ & $4(25,0)$ & $4(25,0)$ & $3(18,7)$ & $3(18,7)$ & $3(18,7)$ \\
\hline AMP & $2(12,5)$ & $3(18,7)$ & $3(18,7)$ & $1(6,3)$ & 0 & 0 \\
\hline AMM & $7(43,7)$ & $4(25,0)$ & $3(18,7)$ & $6(37,5)$ & $7(43,7)$ & $7(43,7)$ \\
\hline DP & $2(12,5)$ & $5(31,3)$ & $6(37,5)$ & $6(37,5)$ & $6(37,5)$ & $6(37,5)$ \\
\hline \multicolumn{7}{|c|}{ Introdução pastoso } \\
\hline Sim & 0 & 0 & 0 & $4(25,0)$ & $6(37,5)$ & $10(62,5)$ \\
\hline Não & $16(100)$ & $16(100)$ & $16(100)$ & $12(75)$ & $10(62,5)$ & $6(37,5)$ \\
\hline \multicolumn{7}{|l|}{ Hábitos orais } \\
\hline Sim & $11(68,7)$ & $10(62,5)$ & $11(68,7)$ & $13(81,3)$ & $12(75,0)$ & $9(56,3)$ \\
\hline Não & $5(31,3)$ & $6(37,5)$ & $5(31,3)$ & $3(18,7)$ & $4(25,0)$ & $7(43,7)$ \\
\hline
\end{tabular}

Legenda: PS= pessoal social; MFA=motor fino adaptativo; $L G G=$ linguagem; $M G=$ motor grosseiro; $A M E=$ aleitamento materno exclusivo; $\mathrm{AMP}=$ aleitamento materno predominante; $\mathrm{AMM}=$ aleitamento materno misto; $\mathrm{DP}=$ desmame precoce.

\section{Discussão}

O AME possui todas as propriedades necessárias e essenciais para a nutrição adequada do lactente até o sexto mês de vida. Associado a isso diversas pesquisas já demostram o efeito positivo do AM evitando mortes infantis, diarreia, infecção respiratória; diminuindo o risco de alergias, de hipertensão, colesterol alto e diabetes; reduzindo a chance de obesidade; apresentando efeito positivo na inteligência e no melhor desenvolvimento da cavidade bucal. Para a mãe, o AM parece estar associado à menor incidência de hemorragia no pós-parto, recuperação mais rápida de seu peso an- terior à gestação, maior espaçamento entre partos, diminuição de risco de certos tumores, menores custos financeiros e promoção do vínculo afetivo entre mãe e filho ${ }^{10}$.

A literatura é controversa sobre influência da idade materna no desmame precoce. Alguns trabalhos consideram que a idade superior a 20 anos é um fator de proteção para o aleitamento materno, ${ }^{11,12}$ enquanto que em outros esse fator não esteve significantemente associado ao início das práticas de desmame precoce ${ }^{13,14}$. Neste trabalho, a média de idade das mães foi razoavelmente elevada, o que pode ser justificado pelos critérios de inclusão do estudo, em que a mãe deveria ter mais de 18 anos, mães adolescentes não participaram desta 
pesquisa, impossibilitando assim a associação da idade materna com o risco de desmame.

O nível de escolaridade da mãe, que muitas vezes é visto como um fator de confusão com a idade, já que quanto mais nova a mãe menor será a sua escolaridade, também é divergente na literatura. Entretanto, a maior parte dos estudos relatam a baixa escolaridade como fator de risco para a amamentação ${ }^{11,13,14}$. Neste sentido, uma publicação do Comitê de Nutrição da Sociedade Europeia de Gastrenterologia, Hepatologia e Nutrição ${ }^{15}$ aponta como fator de proteção ao AM, em países industrializados, o nível educacional e socioeconômico mais elevado, provavelmente pelo maior conhecimento que têm em relação às vantagens do aleitamento materno para a mãe e para a criança. Já em países em desenvolvimento, ocorre o oposto, o que pode ser uma consequência da maior influência das políticas públicas de incentivo ao aleitamento materno sobre a população de baixa renda.

Corroborando com os autores ${ }^{15}$, neste estudo a maioria das mães tinha apenas o ensino fundamental incompleto, ou seja, baixa escolaridade associada como um baixo nível socioeconômico já que a renda familiar média foi inferior a um salário mínimo. Apesar disso, a maioria manteve o aleitamento materno até os seis meses; no entanto, algumas das progenitoras realizaram desmame precoce, mesmo recebendo orientações desde o primeiro mês de vida do lactente, sobre a importância do aleitamento materno. Dentre os motivos mais citados pelas mães, estão: "não tenho leite", "precisava acordar de madrugada para dar leite, pois a criança estava sempre com fome" e "a criança não quer mamar". O grupo que afirmou não ter leite foi orientado a buscar o banco de leite da cidade, no entanto nenhuma delas seguiu a recomendação.

Ainda tratando-se das condições socioeconômicas familiares, autores consideram ${ }^{16}$, a baixa renda um tipo de ameaça constante que aumenta a vulnerabilidade da criança, elevando também a probabilidade da mesma apresentar subnutrição, privação social e futura desvantagem educacional, o que comprometeria o seu desenvolvimento global. O baixo nível socioeconômico do grupo pesquisado não influenciou negativamente o desenvolvimento dos lactentes, tendo em vista que a grande maioria apresentou-se normal ou avançado no sexto mês de vida. Este desempenho pode ser justificado, pelo acompanhamento fonoaudiológico mensal que proporcionou informações aos cuidadores quanto à importância e as formas de estimular a criança, possibilitando um maior desenvolvimento neuropsicomotor infantil. Confirmando os achados do estudo ${ }^{17}$ que considerou o acompanhamento infantil e a construção de laços entre profissional/criança/ família, por meio de uma escuta qualificada, atenta e humanizada, como uma ferramenta na promoção e prevenção da saúde numa perspectiva integral.

Pesquisas demonstram que a cesariana, procedimento realizado pela maioria das mães deste estudo, é um fator que pode comprometer o aleitamento materno levando ao desmame precoce por diversos fatores como a dificuldade no posicionamento do lactente; retardos das respostas endócrinas, pois o procedimento é muitas vezes realizado antes do tempo natural do nascimento. Além disso, o corpo da mãe pode não estar preparado para a produção adequada do leite e o uso de medicamentos, mais frequentes nestes casos, comprometem a prática da amamentação ${ }^{18-20}$.

Apesar disso, todas as mães amamentaram na primeira hora de vida do lactente, o que é considerado essencial para a efetividade do aleitamento materno, além de ser um fator protetivo quanto à mortalidade infantil antes de um ano de vida ${ }^{20}$. O número expressivo de amamentação na primeira hora de vida nesta pesquisa pode estar relacionado à capacitação da equipe da maternidade sobre a prestação de assistência e orientação acerca do aleitamento, já que a instituição faz parte da rede Hospital Amigo da Criança. Essa iniciativa é vista como um importante fator de proteção ao AM e uma estratégia fundamental para a melhoria da saúde da criança ${ }^{10}$. Fato que associado às orientações fonoaudiológicas mensais pode explicar o grande número de mães que mantiveram o AM até o sexto mês.

A introdução precoce de alimentos também pode influenciar na duração do aleitamento materno, interferir negativamente na absorção dos nutrientes do leite materno e provocar reações alérgicas $^{21}$, sendo que algumas práticas, consideradas culturais, mostram-se contrárias às recomendações alimentares oferecidas aos lactantes. A crença de que o leite materno é insuficiente para suprir todas as necessidades do lactente é algo comum na sociedade e que acaba determinando a introdução de substâncias como água, sucos, chás, e, principalmente alimentos artificiais, modificados e complementares na dieta desses lactentes ${ }^{22}$. 
Nesta pesquisa, foi visível a influência deste fator cultural, principalmente quanto à introdução de chás, água e sucos por orientação, principalmente, das avós. O que justificaria a introdução precoce destes líquidos pela maioria das mães ainda no primeiro mês de vida dos lactentes e o baixo índice de AME até o sexto mês, mesmo recebendo orientações de que não era necessário e que poderia influenciar no sucesso do aleitamento materno. Nesses casos, o papel da equipe de fonoaudiologia foi de incentivar o AM, ainda que misto.

Outra função importante, que deve ser discutida, é o papel do AM em suprir as necessidades de sucção que o lactente apresenta. Isto ocorre devido ao esforço muscular exercido durante a mamada, o que não acontece no uso de mamadeira, em que o fluxo de leite é maior fazendo com que o lactente se satisfaça mais rápido e com menor esforço da musculatura. Assim, o êxtase emocional com relação ao impulso da sucção não é atingido, e a criança, para isso procura substituições como o dedo, a chupeta e objetos para satisfazer-se. Desta forma, as crianças que são amamentadas no seio possuem uma menor probabilidade de adquirir hábitos orais deletérios ${ }^{23}$.

No presente estudo, a maioria das crianças que estavam em AM não adquiriram hábitos orais; enquanto que todos os participantes do grupo de DM faziam uso de chupeta. Isso também foi observado em outros estudos, que encontraram uma forte ligação entre aleitamento materno e ausência de hábitos orais deletérios, sendo, inclusive, a chupeta relacionada como fator causal do desmame precoce ${ }^{23-25}$.

Em estudo longitudinal, realizado com crianças chilenas, percebe-se uma associação positiva entre o AM e o melhor desenvolvimento neuropsicomotor, tanto na infância como na vida adulta, pois os nutrientes e componentes do leite materno auxiliam o desenvolvimento do cérebro, além de trazer benefícios biológicos e interacionais entre mãe/lactente ${ }^{26}$. Em outro estudo, observou-se, por meio da aplicação do Denver II, relação entre o desenvolvimento neuropsicomotor com o tempo de amamentação, sendo que o risco para atraso no desenvolvimento foi gradualmente menor, quanto maior foi o tempo da criança em aleitamento materno ${ }^{27}$.

A partir da aplicação mensal do Denver II nos lactentes acompanhados até os seis mês de vida foi possível obter resultados que sugerem uma maior incidência de risco a partir de quatro meses de idade, o que pode estar relacionado à introdução de outros alimentos na dieta da criança e, consequentemente, ao desmame precoce. Entretanto são necessários estudos futuros com um maior número de pacientes e um maior controle das variáveis que podem influenciar o desenvolvimento infantil para generalizar esses achados.

Foi observado, também, que na área do motor grosseiro a maioria dos lactentes apresentava desenvolvimento avançado no sexto mês de vida. $\mathrm{O}$ que pode ser justificado pelo fato de que uma das principais orientações realizadas pelas pesquisadoras durante as visitas foi justamente quanto à inserção da prática do brincar no cotidiano familiar; com sugestões de estratégias de brincadeiras que propiciassem melhor desenvolvimento da criança, estimulando as habilidades motoras, de linguagem e pessoal-social. Como relatado na literatura ${ }^{28}$ os jogos e brincadeiras têm papel importante no desenvolvimento psicomotor, pois estimulam a criatividade, a imaginação, a exploração do corpo no espaço e a manipulação de objetos diversos.

Os resultados positivos desta pesquisa demostram a importância do acompanhamento profissional mensal nos primeiros meses de vida do lactente que com orientações e informações conseguiu que a maioria das mães mantivesse o AM mesmo com a introdução precoce de outros líquidos e, se tornassem mais ativas na estimulação do desenvolvimento neuropsicomotor de seus bebês. Isso confirma os achados da literatura ${ }^{29}$ que observaram que estratégias de acompanhamento após a alta hospitalar, por meio de visitas domiciliares, favorecem as taxas, a iniciação e a duração do AM. Entretanto, ações de incentivo e apoio à amamentação no período pré e pós-natal se concentram apenas em algumas regiões do país, sendo necessário, portanto, uma maior abrangência dentro do Sistema Único de Saúde aumentando a acessibilidade como forma de fortalecer a saúde do bebê e da mãe.

Contudo, a prática do AME nos primeiros seis meses de vida nesta pesquisa, assim como em todo o Brasil, ainda está longe do ideal. Como bem descrito por Granja e Cunha, ${ }^{30}$ é importante que novos estudos busquem fatores como a disponibilidade materna para amamentar e suas justificativas para recusar-se a fazê-lo, fatores da dinâmica familiar que podem favorecer ou não a prática, condições emocionais das mães que influenciam a compreensão das orientações dos profissionais da saúde; 
dentre outros aspectos relevantes articulados a fatores socioculturais e psíquicos, que estão envolvidos no enfrentamento dos dilemas e conflitos inerentes ao aleitamento.

\section{Conclusão}

Os achados desta pesquisa demonstraram que os lactentes que permaneceram em aleitamento materno, mesmo que misto, apresentaram um melhor desenvolvimento neuropsicomotor e uma menor incidência de hábitos orais deletérios, porém não houve diferença quanto à introdução alimentar de líquidos e pastosos.

Além disso, pôde-se observar a importância do apoio às mães com orientações quanto aos benefícios da amamentação e da estimulação do desenvolvimento neuropsicomotor, demostrando que o acompanhamento do lactente deve ser realizado por uma equipe multidisciplinar, com a participação do fonoaudiólogo, permitindo assim uma visão global do desenvolvimento infantil.

\section{Referências}

1. World Health Organization (WHO). Collaborative Study Team on the Role of Breastfeeding on the Prevention of Infant Mortality. Effect of breastfeeding on infant and child mortality due to infectious diseases in less developed countries: a pooled analysis. Lancet. 2000; 355(9202): 451-5.

2. Dee DL, Li R, Lee L, Grummer-Strawn LM. Associations between breastfeeding practices and young children's language and motor skill development. Pediatrics. 2007; 119(1): S92-8.

3. Crestani AH, Souza APR, Beltrami L, Moraes AB Análise da associação entre tipos de aleitamento, presença de risco ao desenvolvimento infantil, variáveis obstétricas e socioeconômicas. J Soc Bras Fonoaudiol. 2012; 24(3): 205-210

4. Silva WF, Guedes ZCF. Tempo de aleitamento materno exclusivo em recém-nascidos prematuros e a termo. Rev CEFAC. 2013; 15(1): 160-171.

5. Brasil. Ministério da Saúde. Secretaria de Atenção à Saúde. Área Técnica de Saúde da Criança e Aleitamento Materno. Rede Amamenta Brasil: os primeiros passos (2007-2010). Brasília: Editora do Ministério da Saúde, 2011.

6. Vargas GS, Alves VH, Rodrigues DP, Branco MBL, Souza RMP, Guerra JVV. Atuação dos profissionais de saúde da estratégia saúde da família: Promoção da prática do aleitamento materno. Revista Baiana de Enfermagem. 2016; 30(2): 1-9.

7. Rodrigues AP, Padoin SMM, Paula CC, Guido LA. Fatores que interferem na autoeficácia da amamentação: Revisão integrativa. Rev enferm UFPE on line. 2013; 7: 4144-52.

8. World Health Organization (WHO). Indicators for assessing infant and young child feeding practices. Conclusions of consensus meeting held 6-8. Washington, 2007.
9. Frankenburg FK, Dodds JB, Archer P, Bresnick B, Maschka P, Edelman N, Shapiro H. Denver II: Technical Manual and Training Manual. Denver: Denver Developmental Materials Inc., 1990

10. Toma TS, Rea MF. Benefícios da amamentação para a saúde da mulher e da criança: um ensaio sobre as evidências. Cad Saúde Pública 2008; 24(2): S235-46.

11. Margotti E, Epifanio M. Aleitamento materno exclusivo e a Escala de Autoeficácia na Amamentação. Rev Rene. 2014; 15(5): 771-9.

12. Carrascoza KC, Possobon RF, Ambrosano GMV, Costa Junior AL, Moraes ABA. Determinants of the exclusive breastfeeding abandonment in children assisted by interdisciplinary program on breast feeding promotion. Ciênc Saúde Coletiva. 2011; 16(10): 4139-46.

13. Salustiano LPQ, Diniz ALD, Abdallah VOS, Pinto RMC. Fatores associados à duração do aleitamento materno em crianças menores de seis meses. Rev. Bras. Ginecol Obstet. 2012; 34(1): 28-33

14. Souza SNDH, Migoto MT, Rossetto EG, Mello DF. Prevalence of breastfeeding and associated factors in the municipality of Londrina (PR, Brazil). Acta Paul Enferm. 2012; 25(1): 29-35.

15. ESPGHAN. Committee on Nutrition, Agostoni C, Braegger C, Decsi T, Kolacek S, Koletzko B et al. Breast-feeding: a commentary by the ESPGHAN Committee on Nutrition. J Pediatr Gastroenterol Nutr. 2009; 49: 112-25.

16. Pilz EML, Schermann LB. Determinantes biológicos e ambientais no desenvolvimento neuropsicomotor em uma amostra de crianças de Canoas/RS. Ciênc. saúde coletiva. 2007; 12(1): 181-90.

17. Silva KD, Araújo KD, Sales LKO, Valença CN, Morais FRR, Morais IF. Acompanhamento do crescimento e desenvolvimento infantil na visão de mães da estratégia saúde da família. Rev bras pesqui saúde. 2014; 16(2): 67-75.

18. Boccolini CS, Carvalho ML, Oliveira MIC, Vasconcellos AG. Factors associated with breastfeeding in the first hour of life. Rev Saúde Pública. 2011; 45(1): 69-78.

19. Hazir T, Akram DS, Nisar YB, Kazmi N, Agho KE, Abbasi $\mathrm{S}$, et al. Determinants of suboptimal breast-feeding practices in Pakistan. Public Health Nutr. 2013; 16(4): 659-84.

20. Pereira CRVR, Fonseca VM, Oliveira MIC, Souza IEO, Mello RR. Avaliação de fatores que interferem na amamentação na primeira hora de vida. Rev. bras. epidemiol. 2013; 16(2): 525-34.

21. Corrêa EM, Corso ACT, Moreira EAM, Kazapi IAM. Alimentação complementar e características maternas de crianças menores de dois anos de idade em Florianópolis (SC). Rev Paul Pediatr. 2009; 27(3): 258-64.

22. Schincaglia RM, Oliveira AC, Sousa LM, Martins KA. Práticas alimentares e fatores associados à introdução precoce da alimentação complementar entre crianças menores de seis meses na região noroeste de Goiânia. Epidemiol Serv Saúde. 2015; 24(3): 465-74.

23. Araújo CMT, Silva GAP, Coutinho SP. Aleitamento materno e uso de chupeta: repercussões na alimentação e no desenvolvimento do sistema sensório motor oral. Rev Paul Pediatria. 2007; 25(1): 59-65. 
24. Miotto MHMB, Caxias FP, Campos DMKS, Ferreira LFPF, Brcellos LA. Aleitamento materno como fator de proteção contra a instalação de hábitos bucais deletérios. Rev CEFAC. 2014; 16: 244-51.

25. Vasconcelos FMN, Massoni ACLT, Heimer MV, Ferreira AMB, Katz CRT, Rosenblatt A. Non-nutritive sucking habits, anterior open bite and associated factors in Brazilian children aged 30-59 months. Braz Dent J. 2011; 22(2): 140-5.

26. Clark KM, Castillo M, Calatroni A, Walter T, Cayazzo M, Pino P, Lozoff B. Breastfeeding and mental and motor development at $5 \frac{1}{2}$ years. Ambul Pediatr. 2006; 6(2): 65-71.
27. Halpern R, Giugliani ERJ, Victora CG, Barros FC, Horta BL. Fatores de risco para suspeita de atraso no desenvolvimento neuropsicomotor aos 12 meses de vida. Rev chil pediatr. 2002; 73(5): 529-39.

28. Scalha, T. B., Souza, V. G., Boffi, T., Carvalho, A. C. The entertainment as resource physiotherapeutic in development psychomotor. Revista de Psicologia da UNESP. 2010; 9(2): 79-92.

29. Costa THM, Sousa LM. Ações de incentivo e apoio a amamentação no período pós-natal no Brasil. Gestão e Saúde. 2013; 4(1): 1600-15.

30. Granja DMA, Cunha MC. Aleitamento materno e artificial ao logo da história: aspectos sócio-culturais. Distrúb Comum. 2011; 23: 237-8. 
ANEXO

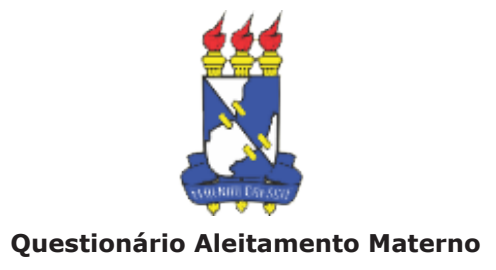

I - Informações pessoais:

Nome:

D.N (mãe):

Idade:

Escolaridade:

Profissão:

Data do parto:

Nome do bebê:

Sexo do bebê:

Endereço para visita:

Telefone:

Datas Visitas (preencher mês a mês, conforme ocorrer).

10:

20

30

40

50

60

II - Dados socioeconômicos (MATERNIDADE)

1- Quantas pessoas moram com você? (incluindo parentes, filhos e amigos)

2- A casa onde você mora é:

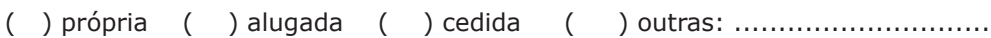

3- Qual a renda FAMILIAR mensal? (somando a renda de todos os que moram na casa)

4- Qual a sua renda?

5- Trabalha fora de casa? Quantas horas? Com quem fica a criança?

6- Situação conjugal:

( )casada ( ) solteira ( ) viúva ( ) mora com o companheiro $($ ) outras:.........

7- Tem quantos filhos:

III - Informações sobre o parto (MATERNIDADE):

8- Tipo de parto: ( ) cesárea ( ) normal

9- Quantas semanas de gestação:

10- Alguma intercorrência?

11- Conseguiu amamentar no peito enquanto estava na Maternidade?

IV - Informações após a alta hospitalar: (aplicado apenas na visita de 1 mês):

13- O bebê fez o teste da orelhinha? (confirmar na carteirinha)

14- Fez algum retorno na Maternidade ou na Unidade de Saúde? Tem algum retorno agendado? (mostrar a carteirinha do bebê- anotar as datas dos retornos e consultas)

$V$ - Informações sobre a saúde do bebê (APLICAR EM TODAS AS VISITAS):

15- Está fazendo uso de algum medicamento?

16- Teve algum episódio de otite (dor de ouvido)?

17- Está com algum outro problema de saúde?

18- Frequenta alguma creche ou escolinha? Desde que idade? 
VI- Dados sobre aleitamento, alimentação e hábitos orais (EM TODAS AS VISITAS):

19- Amamenta no peito? Se não, escrever o porquê.

\begin{tabular}{|c|c|c|c|c|c|}
\hline $1^{\circ}$ mês & $2^{\circ}$ mês & $3^{\circ}$ mês & $4^{\circ}$ mês & $5^{\circ}$ mês & $6^{\circ}$ mês \\
\hline ( ) $\mathrm{S}_{\mathrm{Pq}}($ ) $\mathrm{N}$ & ( ) $\mathrm{S}_{\mathrm{Pq}}($ ) $\mathrm{N}$ & ( ) $\mathrm{S}_{\mathrm{Pq}}($ ) $\mathrm{N}$ & ( ) $\mathrm{S}_{\mathrm{Pq}}($ ) $\mathrm{N}$ & ( ) $\mathrm{S}_{\mathrm{Pq}}($ ) $\mathrm{N}$ & ( ) $\mathrm{S}_{\mathrm{Pq}}($ ) $\mathrm{N}$ \\
\hline
\end{tabular}

20- Introduziu algum outro alimento ou líquido (água, chá, suco)?

\begin{tabular}{|c|c|c|c|c|c|}
\hline $1^{\circ}$ mês & $2^{\circ}$ mês & $3^{\circ}$ mês & $4^{\circ}$ mês & $5^{\circ}$ mês & $6^{\circ}$ mês \\
\hline $\begin{array}{l}\text { ( ) } \mathrm{S}(\text { ( ) } \mathrm{N} \\
\text { Quais: }\end{array}$ & $\begin{array}{l}\text { ( ) } \mathrm{S}(\text { ( ) } \mathrm{N} \\
\text { Quais: }\end{array}$ & $\begin{array}{l}\text { ( ) } \mathrm{S}(\text { ( ) } \mathrm{N} \\
\text { Quais: }\end{array}$ & $\begin{array}{l}\text { ( ) } \mathrm{S}(\text { ( ) } \mathrm{N} \\
\text { Quais: }\end{array}$ & ( ) $\mathrm{S}_{\text {Quais: }}(\mathrm{N}$ & ( ) $\mathrm{S}_{\text {Quais: }}(\mathrm{N}$ \\
\hline Utiliza: & Utiliza: & Utiliza: & Utiliza: & Utiliza: & Utiliza: \\
\hline & $\begin{array}{l}\text { ( ) Mamadeira } \\
\text { (bico) }\end{array}$ & & $\begin{array}{l}\text { ( ) Mamadeira } \\
\text { (bico) }\end{array}$ & $\begin{array}{l}\text { ( ) Mamadeira } \\
\text { (bico) }\end{array}$ & $\begin{array}{l}\text { ( ) Mamadeira } \\
\text { (bico) }\end{array}$ \\
\hline ( ) orto & ( ) orto & ( ) orto & ( ) orto & ( ) orto & ( ) orto \\
\hline ( ) trad & ( ) trad & ( ) trad & ( ) trad & ( ) trad & ( ) trad \\
\hline $\begin{array}{l}\text { Furo: } \\
\text { ( ) original } \\
\text { ( ) aument. }\end{array}$ & $\begin{array}{l}\text { Furo: } \\
\text { ( ) original } \\
\text { ( ) aument. }\end{array}$ & $\begin{array}{l}\text { Furo: } \\
\text { ( ) original } \\
\text { ( ) aument. }\end{array}$ & $\begin{array}{c}\text { Furo: } \\
\text { ( ) original } \\
\text { ( ) aument. }\end{array}$ & $\begin{array}{l}\text { Furo: } \\
\text { ( ) original } \\
\text { ( ) aument. }\end{array}$ & $\begin{array}{l}\text { Furo: } \\
\text { ( ) original } \\
\text { ( ) aument. }\end{array}$ \\
\hline $\begin{array}{l}\text { COPO }(\quad) \\
\text { COLHER ( }) \\
\text { OUTROS: }\end{array}$ & $\begin{array}{l}\text { COPO ( }) \\
\text { COLHER ( }) \\
\text { OUTROS: }\end{array}$ & $\begin{array}{l}\text { COPO }(\quad) \\
\text { COLHER ( }) \\
\text { OUTROS: }\end{array}$ & $\begin{array}{c}\operatorname{COPO}(\quad) \\
\operatorname{COLHER}(\quad) \\
\text { OUTROS: }\end{array}$ & $\begin{array}{c}\text { COPO ( ) } \\
\text { COLHER ( }) \\
\text { OUTROS: }\end{array}$ & $\begin{array}{c}\text { COPO ( ) } \\
\text { COLHER ( }) \\
\text { OUTROS: }\end{array}$ \\
\hline
\end{tabular}

21- Hábitos orais:

\begin{tabular}{|c|c|c|c|c|c|}
\hline $1^{\circ}$ mês & $2^{\circ}$ mês & $3^{\circ}$ mês & $4^{\circ}$ mês & $5^{\circ}$ mês & $6^{\circ}$ mês \\
\hline $\begin{array}{l}\text { ( ) NÃO } \\
\text { ( ) SIM } \\
\text { ( ) DEDO } \\
\text { ( ) CHUPETA } \\
\text { Orto ( }) \\
\text { Tradic. }(\text { ) } \\
(\quad) \text { OUTROS }\end{array}$ & $\begin{array}{c}\text { ( ) NÃO } \\
\text { ( ) SIM } \\
\text { ( ) DEDO } \\
\text { ( ) CHUPETA } \\
\text { Orto ( ) } \\
\text { Tradic. ( }) \\
(\quad) \text { OUTRO }\end{array}$ & $\begin{array}{c}\text { ( ) NÃO } \\
\text { ( ) SIM } \\
\text { ( ) DEDO } \\
\text { ( ) CHUPETA } \\
\text { Orto ( }) \\
\text { Tradic. }(\text { }) \\
(\quad) \text { OUTROS }\end{array}$ & $\begin{array}{c}\text { ( ) NÃO } \\
\text { ( ) SIM } \\
\text { ( ) DEDO } \\
\text { ( ) CHUPETA } \\
\text { Orto ( }) \\
\text { Tradic. ( }) \\
(\quad) \text { OUTROS }\end{array}$ & $\begin{array}{c}\text { ( ) NÃO } \\
\text { ( ) SIM } \\
\text { ( ) DEDO } \\
\text { ( ) CHUPETA } \\
\text { Orto ( ) } \\
\text { Tradic. ( }) \\
(\quad \text { ) OUTROS }\end{array}$ & $\begin{array}{l}\text { ( ) NÃO } \\
\text { ( ) SIM } \\
\text { ( ) DEDO } \\
\text { ( ) CHUPETA } \\
\text { Orto ( }) \\
\text { Tradic. }(\text { ) } \\
(\quad) \text { OUTROS }\end{array}$ \\
\hline
\end{tabular}

